\title{
Analysis of an Adaptive Key Selection Scheme in Wireless Sensor Networks
}

\author{
Guorui $\mathrm{Li}^{1}$, Jingsha $\mathrm{He}^{2}$, and Yingfang $\mathrm{Fu}^{1}$ \\ ${ }^{1}$ College of Computer Science and Technology \\ Beijing University of Technology \\ Beijing 100022, China \\ \{liguorui, fmsik\}@emails.bjut.edu.cn \\ ${ }^{2}$ School of Software Engineering \\ Beijing University of Technology \\ Beijing 100022, China \\ jhe@bjut.edu.cn
}

\begin{abstract}
Sensor networks are suitable for a variety of commercial and military applications due to their self-organization characteristics and distributed nature. As a fundamental requirement for security functionality in sensor networks, key management plays a central role in authentication and encryption. In this paper, we describe an Adaptive Key Selection (AKS) scheme for multi-deployment in sensor networks and analyze the scheme in the aspects of security, connectivity and overhead. Our analysis shows that the AKS scheme can greatly improve the connectivity of sensor nodes while maintaining the security of an existing multi-deployment scheme.
\end{abstract}

Keywords: sensor networks, security, key predistribution, multi-deployment.

\section{Introduction}

Recent advances in micro-electro-mechanical systems, electronics and wireless communications have made it practical now to develop and deploy low-cost, high-performance and low-power sensor nodes. These nodes are equipped with sensing, processing and communication capabilities. In such a network, security is important to guarantee confidentiality, integrity and availability of transported data. As the basic requirement for security functionality, key management plays a central role in data encryption and in authentication. However, due to energy and resource limitations in sensor nodes, many ordinary security mechanisms are deemed impractical, if not infeasible, in sensor networks.

There are currently three types of key management schemes that are commonly used in sensor networks: trusted server scheme, self-enforcing scheme, and key predistribution scheme. The first type of scheme, i.e., the trusted server scheme, relies on a trusted server for key distribution and management. The second type of scheme, i.e., the self-enforcing scheme, relies on asymmetric cryptography for key distribution and management using public key certificates. However, the lack of a trusted infrastructure in application environments and limited computation and energy resources in sensor nodes make these two types of schemes undesirable. The third type of scheme, i.e., the 
key predistribution scheme, is one in which cryptographic keys are predistributed in all sensor nodes prior to deployment [1]. There are already several key predistribution schemes that have been proposed, such as the basic probabilistic key predistribution scheme [2], the q-composite key predistribution scheme [3], the random pairwise key scheme [3], the random subset assignment scheme [4], the grid-based key predistribution scheme [4], the closest pairwise key predistribution scheme [5], and the closest polynomials predistribution scheme [5]. The Adaptive Key Selection scheme is developed based on the above basic schemes.

As time goes by, some sensor nodes may be destroyed, compromised or dead. Since these nodes no longer work properly, the sensor network may become fragmented. The consequence is that not all collected data can be transmitted to the sink node that collects the data. Therefore, new sensor nodes have to be deployed in the network to replace those out-of-function nodes to reestablish a connected network. However, little work has been done so far to address this concern.

One simple method is to deploy a group of new sensor nodes that are loaded with predistributed keys selected from the same key pool as that for the previously deployed set of sensor nodes. We call this scheme the basic multi-deployment scheme. The benefit of this scheme is that the newly deployed sensor nodes share the same group of predistributed keys as those for the previously deployed sensor nodes so that they can establish connections fairly easily. However, its shortcoming is equally obvious. That is, the sensor nodes that have already been compromised will have negative impact on the newly deployed sensor nodes. As the result, the newly deployed sensor nodes may not be safe from the very beginning of their deployment when the percentage of the compromised sensor nodes exceeds a certain number.

Arjan Durresi et al. proposed the Secure Continuity for Sensor Networks (SCON) scheme [6]. In this scheme, the sensor nodes that belong to different deployment sets are loaded with predistributed keys from different key pools. And the bridge nodes with large memory and high computation power are deployed at the same time to help the sensor nodes establish secure links between the sensor nodes in the new deployment set and those in the previous deployment set. However, the probability of establishing a secure link between any two sensor nodes that belong to two different deployment sets is very low because the bridge node is only loaded with predistributed keys that are randomly selected from the key pool of the new deployment set and that of the previous deployment set. Therefore, extra help is needed from actors that possess arbitrary moving capacity so that they can be deployed in lower connectivity areas to help sensor nodes establish secure links.

In this paper, we describe an Adaptive Key Selection (AKS) scheme for multi-deployment in sensor networks. This scheme can be applied to the hierarchical wireless sensor networks in multiple deployments of sensor nodes. We use three types of network elements in this scheme: base station, cluster head nodes and ordinary sensor nodes. We assume that the base station stores all the predistributed keys for every sensor node and the cluster head nodes have strong computation, memory and communication power and can communicate with the base station using an asymmetric encryption algorithm. Every cluster head node executes the AKS algorithm to select the optimal key set and assists in establishing secure links between any two sensor nodes that belong to two different deployment sets. Our analysis shows that our scheme can greatly improve the connectivity between any two sensor nodes that belong to two 
different deployment sets. The flexibility of the AKS scheme ensures that it can be combined with any key predistribution scheme described in [1-5].

The rest of the paper is organized as follows. In the next section, we describe the AKS scheme. In Section 3, we analyze the connectivity, security and overhead aspects of this scheme. In Section 4, we identify some related work in sensor network security. Finally, in Section 5, we conclude this paper and discuss some future research directions.

\section{The Adaptive Key Selection Scheme}

In the AKS scheme, all sensor nodes in the network are classified into three different types: the base station, the cluster head nodes and the ordinary sensor nodes. These nodes perform different functions to achieve the goal, which is described in this section.

\subsection{Key Determination in the Cluster Head Nodes}

The strong computation, memory and communication power of the cluster head nodes enable these nodes to use an asymmetry encryption algorithm to secure communication with the base station node. Each cluster head node operates by following the procedure below:

(1) A cluster head node broadcasts a query message to acquire the ID information of its neighboring sensor nodes.

(2) After receiving a query message, a sensor node transmits its ID information to the cluster head node.

(3) The cluster head node collects the ID information from the neighboring sensor nodes, encrypts such information in a message using its private key $K_{p r i}$ and sends the message to the base station. The base station verifies the message using the cluster head node's public key and retrieves the ID information.

(4) The base station encrypts the predistributed keys for the sensor nodes contained in the message using the cluster head node's public key $K_{p u b}$ and sends the resulting message to the cluster head node.

(5) The cluster head node decrypts the received message from the base station using its private key and gets the predistributed keys for its neighboring sensor nodes.

(6) The cluster head node runs the AKS algorithm presented below to select the optimal key set.

\subsection{The Adaptive Key Selection (AKS) Algorithm}

The Adaptive Key Selection algorithm works as follows:

(1) For all the sensor nodes, $S_{l}, \ldots, S_{m}$, that belong to the same deployment set, we build a $n \times m$ matrix $M$ to describe key predistribution status where $n$ is the number of different keys and $m$ is the number of sensor nodes. We set $M_{i j}=1$ if sensor node $S_{i}$ is predistributed with the $j$ th key, otherwise, $M_{i j}=0$.

(2) We sum the matrix $M$ by column and the resulting $n \times 1$ vector $V$ represents the number of each predistributed key in the deployment set. 
(3) We select the maximum element in vector $V$ and the corresponding row number $i_{\max }$ is the number of the optimal key selected in this round. If there is more than one such maximum element, we just select the first such element.

(4) We set all the elements of column $j$ to be 0 if $M_{\text {imaxj }}=1$ to exclude the nodes that hold the selected optimal key.

(5) Repeat steps (2)-(4) until all the elements in $M$ become 0.

When the algorithm completes, we have the queue for optimal key selection for the deployment set. All the keys in this queue are critical keys and they form the minimum spanning set of all the predistributed keys for the deployment set. The closer the position of a key to the front of the queue, the higher the number of sensor nodes that share this key, and this key should, therefore, be selected with a higher priority.

\subsection{Key Establishment in Ordinary Sensor Nodes}

In an ordinary sensor node, key establishment includes three phases: (1) key predistribution phase, (2) direct key establishment phase and (3) path key establishment phase. Two sensor nodes that belong to two different deployment sets can establish a secure path key with the help of the cluster head node. The cluster head node can certainly do so for two sensor nodes in the same deployment set because it stores all the critical keys for this deployment set. If it stores all the critical keys for different deployment sets, we can see that the length of any path key is no more than two hops.

\section{Analysis}

We analyze the AKS scheme in terms of security, connectivity and overhead to demonstrate its feasibility. In particular, we compare the AKS scheme with the SCON scheme in the analysis where appropriate to show that the former is more advantageous over the latter in some key aspects of multi-deployment in sensor networks.

\subsection{Security Analysis}

Fig. 1 shows the relationship between the percentage of compromised links between sensor nodes in a new deployment and that of compromised nodes in the previous deployment in the basic multi-deployment scheme [6]. We can thus see that the

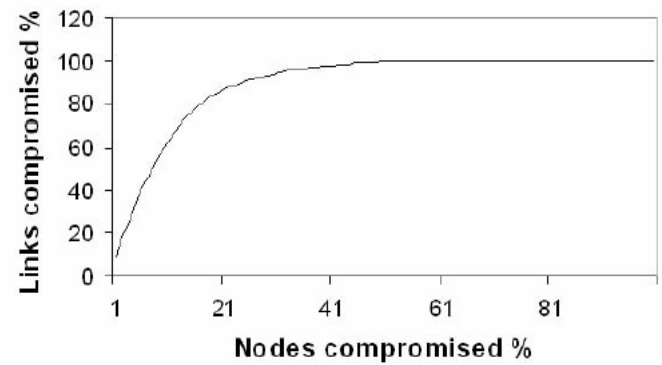

Fig. 1. Relationship between the percentage of compromised links and that of compromised nodes in the basic multi-deployment scheme 
effectiveness of a new deployment of sensor nodes is heavily influenced by the number of compromised sensor nodes in the previous deployment set. In the SCON and the AKS schemes, however, the sensor nodes of a new deployment set are immune to the compromised sensor nodes of the previous deployment set, for the new nodes are loaded with keys predistributed from a different key set. That is, the percentage of compromised links in the SCON and the AKS scheme is 0 .

\subsection{Connectivity Analysis}

In the SCON scheme, the number of keys that are randomly selected from the new deployment set and predistributed into the bridge node is the same as that from the previous deployment set. Therefore, the probability of establishing a link between any two sensor nodes that belong to two different deployment sets is:

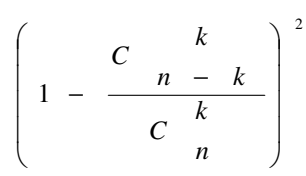

where $n$ is the size of key predistribution pool and $k$ is the number of predistributed keys in each sensor node. And the bridge node is predistributed with $2 k$ number of keys.

In the AKS scheme, the probability of establishing a link between two senor nodes that belong to two different deployment sets is higher than that in the SCON scheme. This is because the keys stored in the cluster head node are selected from the optimal key selection queues of the respective deployment sets. To validate our claim, we did some simulation in which we used 200 as the number of sensor nodes for each deployment set and 1,000 as the size of the pool for predistributed keys. Each sensor node would be predistributed with $k$ keys, where $k=0, \ldots, 100$, that were randomly selected from the key pool. Fig. 2 shows that connectivity between any two sensor nodes that

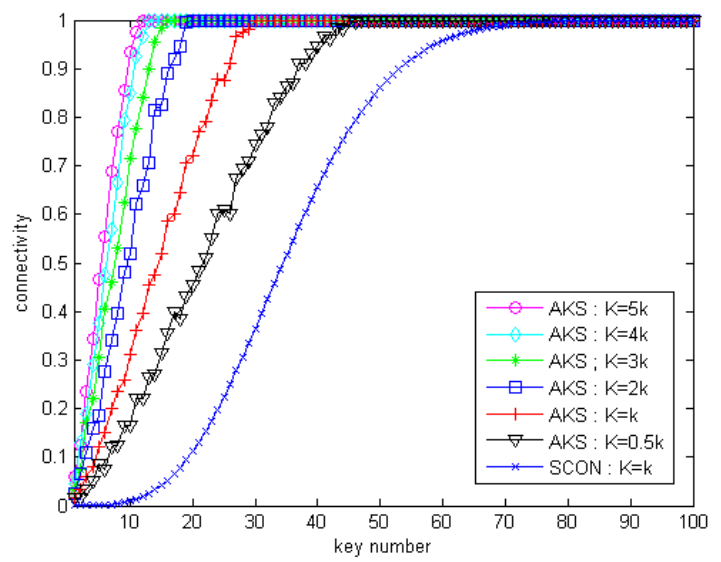

Fig. 2. The connectivity between sensor nodes that belong to two different deployment sets in the SCON and the AKS schemes, respectively 
belong to two different deployment sets in the SCON scheme and that in the AKS scheme. We can thus conclude that network connectivity using the AKS scheme is much better than that using the SCON scheme.

Fig. 3 shows the relationship between connectivity among sensor nodes that belong to two different deployment sets in the AKS scheme and the number of keys $k$ in each sensor node. It also shows the result for different $K$, the number of keys stored in a cluster head node. We can thus see that the higher the number of keys $K$ is in a cluster head node, the higher the connectivity is between any two sensor nodes. We can also see that even when the number of keys in a cluster head in the AKS scheme is only half of that in the SCON scheme, the connectivity between any two sensor nodes is much higher in the AKS scheme than that in the SCON scheme.

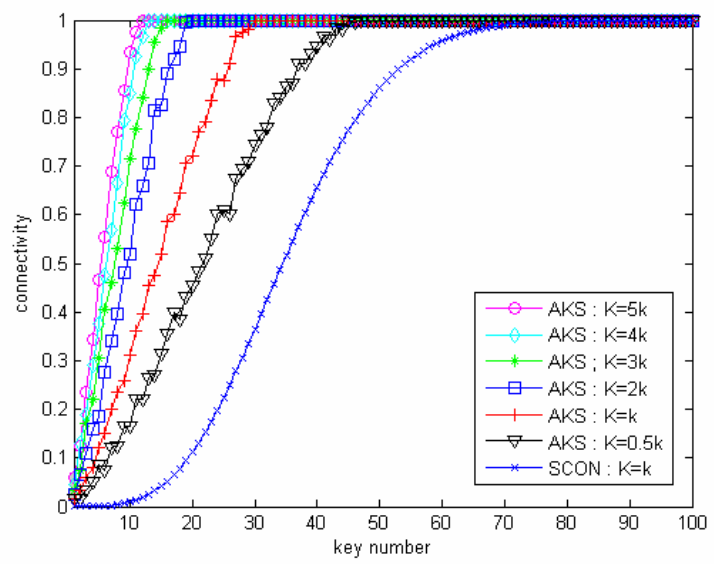

Fig. 3. The connectivity between sensor nodes that belong to two different deployment sets in the AKS scheme for different key storage sizes in the cluster head nodes

\subsection{Overhead Analysis}

The AKS scheme would incur communication and computational overhead to the cluster head nodes while having little impact on the ordinary sensor nodes. The communication overhead mainly results from key request and reply messages between the cluster head nodes and the base station node, and the computational overhead mainly results from encryption and decryption computation in the cluster head nodes for requesting and receiving keys from the base station node as well as from optimal key selection in the AKS algorithm. That is why we require that the cluster head nodes have the necessary computation, storage and communication power.

In order to decrease the communication overhead and the computational overhead, we can predistribute all the keys in every deployment set to the cluster head nodes before the actual deployment. After deployment, the cluster head nodes can collect the ID information of its neighboring sensor nodes and only reserve the optimal keys selected using the AKS algorithm while removing all the other keys to enhance the security of key management [7]. 


\section{Related Work}

Nowadays, there are many studies in the area of security in wireless sensor networks. These studies are mostly focused on key management, authentication, and vulnerability analysis. In addition to studies on key predistribution schemes [1-6], intrusion detection system (IDS) is also very important to detect compromised sensor nodes and ensure the security of the whole network [8, 9]. Furthermore, Wood and Stankovic identified a number of DoS attacks in sensor networks [10] and Deng et al. described a path-based DoS attack and proposed a solution using one-way hash chains to protect end-to-end communication against this type of DoS attacks [11].

\section{Conclusion and Future Work}

In this paper, we described an Adaptive Key Selection scheme and the corresponding algorithm for multi-deployment in sensor networks. Our analysis shows that the AKS scheme can greatly improve the connectivity of sensor nodes while maintaining the security in existing multi-deployment schemes. In the future, we will focus on developing methods to protect the cluster head nodes and to detect compromised sensor nodes in order to further improve the security of sensor networks.

\section{References}

1. Du, W.L., Deng, J., Han, Y.S., Chen, S., Varshney, P.K.: A Key Management Scheme for Wireless Sensor Networks Using Deployment Knowledge. Proc. IEEE INFOCOM 2004. (2004) 586-597

2. Eschenauer, L., Gligor, V.D.: A Key-Management Scheme for Distributed Sensor Networks. Proc. 9th ACM Conference on Computer and Communications Security. (2002) 41-47

3. Chan, H., Perrig, A., Song, D.: Random Key Predistribution Schemes for Sensor Networks. Proc. IEEE Symposium on Research in Security and Privacy. (2003) 197-213

4. Liu, D.G., Ning, P.: Establishing Pairwise Keys in Distributed Sensor Networks. Proc. 10th ACM Conference on Computer and Communications Security. (2003) 52-61

5. Liu, D.G., Ning, P.: Location-based Pairwise Key Establishments for Static Sensor Networks. Proc. 2003 ACM Workshop on Security in Ad Hoc and Sensor Networks. (2003) 72-82

6. Durresi, A., Bulusu, V., Paruchuri, V., Barolli, L.: Secure and Continuous Management of Heterogeneous Ad Hoc Networks. Proc. 20th International Conference on Advanced Information Networking and Applications. (2006) 511-516

7. Simplot-Ryl, D., Simplot-Ryl, I.: Connectivity Preservation and Key Distribution in Wireless Sensor Networks Using Multi-deployment Scheme. Proc. 3rd International Conference on Ubiquitous Intelligence and Computing. (2006) 988-997

8. Silva, A., Martins, M., Rocha, B., Loureiro, A., Ruiz, L., Wong, H.: Decentralized Intrusion Detection in Wireless Sensor Networks. Proc. 1st ACM International Workshop on Quality of Service \& Security in Wireless and Mobile Networks. (2005) 16-23 
9. Roman, R., Zhou, J.Y., Lopez, J.: Applying Intrusion Detection Systems to Wireless Sensor Networks. Proc. 3rd Consumer Communications and Networking Conference. (2006) 640-644

10. Wood, D., Stankovic, J.A.: Denial of Service in Sensor Networks. IEEE Computer, Vol. 35, No. 10. (2002) 54-62

11. Deng, J., Mishra, S.: Defending Against Path-based DoS Attacks in Wireless Sensor Networks. Proc. 3rd ACM workshop on Security of Ad hoc and Sensor Networks. (2005) 89-96 